\title{
IL RUOLO DEL GIUDICE IN RAPPORTO ALL'EVOLUZIONE DEL SISTEMA DELLE FONTI ED ALLA DISCIPLINA DELL'ORDINAMENTO GIUDIZIARIO
}

\author{
ROBERTO ROMBOLI ${ }^{*}$
}

\begin{abstract}
RESUMO: O estudo versa sobre o papel do juiz no Direito italiano, em relação à evolução do sistema das fontes e a disciplina do ordenamento judiciário. Examina-se o tema tendo em vista não somente o desenvolvimento do Direito Constitucional italiano - em face da atuação da Corte Constitucional -, como também do ordenamento comunitário e da legislação ordinária. Procura-se, em essência, situar a função atual do juiz no sistema jurídico italiano, especificar os limites da atividade judicial, em especial no que concerne a do legislador. PALAVRAS-CHAVE: Atividade judicial; Direito italiano; Direito constitucional.
\end{abstract}

ABSTRACT: This study concerns the judge's role in Italian Law, as related to the evolution of the System of Sources and to the matter of judicial regulation. The topic is dealt with attention not only to the development of Italian Constitutional Law - resulting from the work of the Constitutional Court - but also to Communitarian Law and national legislation. The aim is, in essence, to establish the judge's current function in the Italian legal system, to specify the limits of judicial activity, especially when that of the legislator is concerned. KEYWORDS: Judicial activity; Italian Law; Constitutional Law.

SUMÁRIO: 1. Premissas; 2. O papel do juiz em relação à entrada em vigor da Constituição e da atuação da Corte constitucional. A evolução do modelo de ordenamento judiciário; 3. A valorização e os limites da atividade interpretativa do juiz. A formação de um modelo italiano de ordenamento judiciário; 4 . $O$ papel do juiz em relação à concretização do ordenamento comunitário: a pré-judicialidade comunitária e o poder de desaplicar a lei nacional; 5. A produção do direito por parte do legislador e por parte do juiz: forma, modalidade e legitimação; 6 . O papel atual do juiz e a Lei n ${ }^{\circ} 150$ de 2005 de reforma do ordenamento judiciário.

SUMMARY: 1. Premises; 2. The judge's role in relation to the Constitution's coming into force and to the Constitutional Court's activity. The evolution of the judicial regulation model; 3 . The increased valuation and the limits of the judge's interpretive activity. The settling of an Italian model of judiciary regulation; 4. The judge's role in relation to the fulfillment of the Communitarian regulation: the Communitarian pre-judiciality and the power to not apply national legislation; 5 . The production of Law by the legislator and by the judge: form, model and legitimacy; 6 . The judge's current role and statute no. 150 of 2005 which reforms the judiciary regulation.

\footnotetext{
* Catedrático de Direito Constitucional. Professor da faculdade de direito da Universidade de Pisa, Departamento de Direito Público.
} 
SOMMARIO: 1 . Premessa. - 2. Il ruolo del giudice a seguito dell'entrata in vigore della Costituzione e dell'attuazione della Corte costituzionale. L'evoluzione del modello di ordinamento giudiziario - 3. Segue: la valorizzazione ed i limiti dell'attività interpretativa del giudice. La formazione di un modello italiano di ordinamento giudiziario. -4 . Il ruolo del giudice a seguito della realizzazione dell'ordinamento comunitario: la pregiudizialità comunitaria ed il potere di disapplicazione della legge nazionale. -5 . La produzione del diritto da parte del legislatore e da parte del giudice: forme, modalità e legittimazione. 6. Il ruolo attuale del giudice e la legge n. 150 del 2005 di riforma dell'ordinamento giudiziario.

\section{PREMESSA}

Traendo spunto dalla recente approvazione della legge-delega che introduce profonde e sostanziali modifiche alla disciplina dell'ordinamento giudiziario, vorrei svolgere alcune riflessioni in ordine all'incidenza ed alle interrelazioni che si pongono tra la stessa disciplina ed il ruolo che il giudice è venuto assumendo nell'evoluzione del sistema delle fonti del diritto.

Il tema richiama immediatamente il noto principio della subordinazione del giudice alla legge, che la nostra Costituzione esprime all'art. 101, $2^{\circ}$ comma, attraverso l'affermazione secondo cui “i giudici sono soggetti soltanto alla legge”, una formula che più di ogni altra, come esattamente rilevato da Gustavo Zagrebelsky" ${ }^{1}$, risente però nel suo significato delle caratteristiche generali dell'ordinamento giuridico in cui i giudici sono concretamente chiamati ad operare.

$\mathrm{Al}$ proposito concentrerò la mia attenzione su due momenti della evoluzione e modificazione del sistema delle fonti che, a mio giudizio, hanno avuto maggiore influenza sul ruolo attuale del giudice nell'applicazione ed interpretazione del diritto, vale a dire l'approvazione e l'entrata in vigore della Costituzione, da un lato e la progressiva realizzazione dell'ordinamento comunitario, dall'altro.

Se appare indubbio come il ruolo del giudice dipenda dal sistema delle fonti nel quale egli è chiamato a svolgere la sua funzione, credo però sia altrettanto certa l'influenza determinata in proposito da una particolare fonte del diritto, vale a dire la legge sull'ordinamento giudiziario, per la parte in cui disciplina elementi essenziali della vita professionale del magistrato (valutazione delle attitudini, “carriera”, responsabilità, organizzazione del lavoro ecc.), la cui regolamentazione in un modo e nell'altro non può non avere riflessi e condizionamenti sulla realizzazione di un determinato ruolo assegnato al giudice dall'ordinamento. In altri termini la posizione riconosciuta al giudice nel sistema costituzionale ed istituzionale deve trovare una adeguata e conforme risposta nella legge sull'ordinamento giudiziario, la quale è chiamata ad esercitare in proposito una funzione di estrema importanza.

Ciò è stato del resto ben presente ai nostri Costituenti, i quali nel titolo IV della parte seconda della Costituzione, relativo alla magistratura - pur scartando l'ipotesi di fissare in materia una riserva di legge costituzionale o di richiedere una particolare

${ }^{1}$ G. ZAGREBELSKY, Il diritto mite, Torino, 1992, 206, secondo cui tale principio continua comunque ad essere inteso nel senso del servizio passivo della volontà del legislatore, senza tentarne interpretazioni più conformi al senso che ha oggi il legame tra la legge e la sua attuazione attraverso la giurisdizione. 
maggioranza per la sua approvazione - hanno operato in molte occasioni un rinvio alla legge sull'ordinamento giudiziario, quale legge organica della materia, sottolineandone così l'importanza per la realizzazione dei principi costituzionali stabiliti con riferimento alla posizione ed all'attività della magistratura. Importanza sottolineata anche dalla previsione contenuta nella VII disposizione transitoria, dove si richiama la necessità che il futuro parlamento approvi una nuova legge sull'ordinamento giudiziario, in conformità alla Costituzione ${ }^{2}$.

In considerazione di ciò cercherò di sottolineare, seppure in maniera necessariamente sintetica, come al mutamento del ruolo riconosciuto al giudice dall'ordinamento si sia, di pari passo, riscontrata un'analoga e parallela evoluzione della legge (o delle leggi) sull'ordinamento giudiziario, in qualche misura specchio e condizione della posizione del giudice nel sistema.

Questo parallelo mi consentirà al termine di questo intervento di svolgere alcune brevi considerazioni a proposito della recente legge approvata in materia dal parlamento dopo essere stata rinviata alle camere dal presidente Ciampi, il quale aveva rilevato, per alcuni aspetti, una palese violazione della Costituzione ${ }^{3}$.

\section{IL RUOLO DEL GIUDICE A SEGUITO DELL'ENTRATA IN VIGORE DELLA COSTITUZIONE E DELL’ATTUAZIONE DELLA CORTE COSTITUZIONALE. L'EVOLUZIONE DEL MODELLO DI ORDINAMENTO GIUDIZIARIO.}

Con riferimento al primo dei due momenti dell'evoluzione del sistema delle fonti cui facevo riferimento (l'entrata in vigore della Costituzione), essa, in considerazione delle caratteristiche che la contraddistinguono, produce quella che è stata definita una vera e propria "rivoluzione", rispetto all'ordinamento precedente. Nello stato liberale monoclasse, com'è noto, il diritto si esprime essenzialmente e quasi esclusivamente attraverso la legge formale del parlamento, che costituisce l'unica vera e più importante fonte del diritto. La stessa, in quanto indirettamente espressione della sovranità popolare, viene anche in qualche misura considerata per definizione giusta ed è per ciò che non viene previsto alcun sistema di controllo sulle leggi ${ }^{4}$. Riguardo quindi alla giurisdizione vale nettamente l'assoluto primato della legge ed il giudice è conseguentemente visto come un soggetto che, in considerazione delle proprie capacità tecnico-giuridiche, è chiamato ad applicare la stessa, ricercando la volontà con essa espressa dal legislatore. Un giudice, come si dice, "bocca della legge” e meccanico applicatore di un sistema giuridico chiaro e completo.

\footnotetext{
${ }^{2}$ L'importanza della legge è rilevata dal presidente della repubblica Ciampi il quale, nel rinviare alle camere la legge di riforma dell'ordinamento giudiziario, ha giustificato la particolare attenzione prestata alla verifica della costituzionalità della medesima osservando come essa, "preordinata com'è a dare attuazione alla VII disposizione transitoria, $1^{\circ}$ comma, Cost., rappresenta un atto normativo di grande rilievo costituzionale e di notevole complessità".

${ }^{3}$ Nel senso che il Presidente ha inteso segnalare soprattutto i vizi maggiormente di sistema: quelli che cioè manifestavano una sorta di difetto di sensibilità istituzionale del legislatore, v. LUCIANI, Il rinvio presidenziale, in AA.VV., La legge di riforma dell'ordinamento giudiziario, in Foro it., 2006, V, fasc. 1.

${ }^{4}$ In proposito v., di recente, la riflessione di CAVINO, Interpretazione discorsiva del diritto. Saggio di diritto costituzionale, Milano, 2004, spec. 243 ss.
} 
A questo ruolo assegnato al giudice risulta confacente una legge sull'ordinamento giudiziario, che vedeva una magistratura organizzata al suo interno in maniera simile alla pubblica amministrazione, con giudici-funzionari ed un'organizzazione interna di tipo gerarchico, per gradi. Una carriera fatta attraverso concorsi, con i quali selezionare i soggetti tecnicamente più bravi, la presenza di scarse garanzie di autonomia e di indipendenza, sia sotto il profilo della indipendenza esterna che di quella interna. Un sistema di organizzazione quindi in coerenza con la concezione di un giudice meccanico applicatore della legge.

La "rivoluzione" apportata dalla Costituzione non deriva tanto dal fatto che una nuova fonte viene ad inserirsi nel previgente sistema delle fonti, quanto dalle caratteristiche di questo nuovo tipo di fonte: una fonte gerarchicamente sovraordinata rispetto alla legge (rigidità) ed organizzata più per principi che per regole.

La previsione della superiorità gerarchica della Costituzione, e quindi di limiti invalicabili per il legislatore ordinario, comporta che, a differenza del periodo pre-costituzionale orientato per l'assoluta prevalenza del momento "politico" della formazione del diritto, risulta adesso decisiva, per il rispetto di quei limiti e quindi per l'attuazione dei principi costituzionali, l'attività dei giudici, in stretto raccordo con quella della Corte costituzionale.

Tali principi debbono servire, da un lato, a svolgere una funzione unificante per l'interpretazione delle singole leggi spesso espressione di interessi particolari e, dall'altro, a guidare il giudice nell'individuazione della soluzione specifica per il singolo caso di fronte alla difficoltà della legge di prevedere e regolare i casi concreti. Ne deriva un'indubitabile dilatazione della funzione di interpretazione della Costituzione da parte del giudice che fa definitivamente tramontare la visione di questo come "bocca della legge” o come meccanico applicatore di una regola già interamente contenuta e presente nel dettato normativo.

Come scrive Livio Paladin, con l'entrata in vigore della Costituzione "svanisce l'idea del sistema normativo bello e fatto, ontologicamente dato e quindi preesistente rispetto al momento interpretativo" e "subentra, in suo luogo, la realistica visione di un sistema 'in movimento' soggetto a continue evoluzioni: dipendenti non solo dal sopravvenire di nuove discipline, atte a spostare il senso e la portata delle stesse discipline relative ad altre materie o branche dell'ordinamento, ma dall'intrinseco mutare degli indirizzi interpretativi e applicativi, pur fermi restando gli iniziali disposti della Costituzione e delle leggi"

Similmente è stato sottolineato da più parti ${ }^{6}$ come è attraverso il richiamo alla Costituzione ed ai principi innovatori in essa contenuti - la Grande trasformazione del ruolo del diritto nelle moderne società del benessere, di cui parla Mauro Cappelletti ${ }^{7}$ che si realizza la c.d. rivolta contro il formalismo, ossia contro un'interpretazione formale

\footnotetext{
${ }^{5}$ PALADIN, Le fonti del diritto italiano, Bologna, 1996, 110.

${ }^{6}$ Cfr., tra gli altri, TREVES, Giustizia e giudici nella società italiana, Bari, 1972, 155 ss.; DOGLIANI, Interpretazioni della Costituzione, Milano, 1982, 29 ss.; REBUFFA, La funzione giudiziaria, Torino, 1988, 127 ss.

${ }^{7}$ CAPPELLETTI, Giudici legislatori?, Milano, 1984, 22 ss.
} 
del diritto rifacentesi all'esigenza di certezza del diritto ed a favore del riconoscimento dell'attività creativa dell'interpretazione con apertura verso la tutela di nuove situazioni emerse successivamente all'entrata in vigore dell'atto normativo interpretato.

Specie di fronte ai diritti sociali, a domande che vengono poste al giudice per la concreta realizzazione degli stessi, questo vede considerevolmente ampliati gli spazi interpretativi nel fare riferimento e dare applicazione ai principi costituzionali.

L'intuizione di Piero Calamandrei in ordine alla Carta costituzionale come fondamento di "una nuova teoria dell'interpretazione delle leggi", non viene immediatamente recepita dalla magistratura la quale, sulla base della VII disposizione transitoria della Costituzione, era pure chiamata a svolgere una sorta di controllo diffuso di costituzionalità delle leggi, in attesa dell'entrata in funzione della Corte costituzionale. I giudici, e quelli della cassazione in particolare, tendono infatti a mantenere una separazione tra il piano della legalità e quello della costituzionalità ${ }^{8}$, svalutando il significato ed il valore della Costituzione come fonte del diritto, direttamente applicabile da parte del giudice.

Sintomatica in proposito la scelta operata tra l'effetto di abrogazione e quello di incostituzionalità che si sarebbe verificato a seguito dell'entrata in vigore della Costituzione nei riguardi delle leggi anteriori. La giurisprudenza della cassazione si orienta com'è noto nel primo senso, venendo così in qualche modo a considerare la Costituzione come lex posterior più che come lex superior e riconoscendo l'effetto abrogativo solo in presenza di una chiara incompatibilità tra la disposizione costituzionale e quella legislativa. Di conseguenza viene riconosciuta diretta applicazione solo alle disposizioni costituzionali aventi un contenuto specifico, mentre la si nega per quelle contenenti principi, ritenute come tali rivolte solo al potere legislativo (affinché vi dia attuazione) e non a quello giurisdizionale. Nasce da queste posizioni la nota distinzione, operata dalla corte di cassazione, tra norme costituzionali precettive e programmatiche.

A livello organizzativo, nonostante alcune significative riforme (specie quella del 1946), la situazione della magistratura resta sostanzialmente quella del periodo precedente, anche perché non viene ancora attuato l'istituto che contribuirà in maniera notevole all'evoluzione dell'ordinamento giudiziario del nostro paese, ossia il Consiglio superiore della magistratura, che sarà istituito solamente a partire dal 1958.

Un momento di grande rilievo, per il tema che stiamo trattando, è invece rappresentato dall'entrata in funzione nel 1956 della Corte costituzionale, la quale è inevitabilmente subito chiamata a prendere posizione in ordine al problema relativo al rapporto tra i due piani cui ho prima accennato: quello della legalità e quello della costituzionalità. In concreto, a fronte di una questione di legittimità costituzionale sollevata da un giudice nel corso di un giudizio, la Corte costituzionale deve decidere se debba ritenersi di esclusiva competenza del giudice comune l'interpretazione della legge e quindi essa debba limitarsi ad assumere la stessa così come interpretata dal giudice oppure se essa possa a sua volta interpretare la legge in maniera eventualmente

${ }^{8}$ LUCIANI parla in proposito, in un recente scritto (Su legalità costituzionale, legalità legale e unità dell'ordinamento, in Studi in onore di Gianni Ferrara, Torino, 2005, II, 501 ss.) di "legalità legale" e di "legalità costituzionale".

DIREITOS FUnDAMENTAIS E JUSTIÇA N 6 - JAN./MAR. 2009 
diversa rispetto a quella fatta propria dal giudice. Con ciò superando la distinzione dei piani (al giudice la legge, alla Corte la Costituzione, secondo l'espressione di Carlo Mezzanotte) $)^{9}$.

La Corte costituzionale, fin dalle prime decisioni, mostra di accogliere con fermezza la seconda soluzione, come mostrano inequivocabilmente le pronunce interpretative di rigetto, attraverso le quali la Corte dichiara non fondata la questione di costituzionalità, ma fornisce al contempo una lettura interpretativa della stessa tale da evitare la dichiarazione di incostituzionalità. La legge in altri termini viene "salvata" dalla incostituzionalità, a condizione che il giudice segua l'interpretazione indicata dalla Corte come l'unica costituzionalmente conforme.

Questa posizione del Giudice costituzionale produce una reazione da parte soprattutto della Corte di cassazione, la quale si sente in qualche misura espropriata del potere di interpretazione e di nomofilachia, dando luogo a quella che è stata definita la "guerra tra le due corti", poi felicemente superata anche grazie alla valorizzazione, da parte della Corte costituzionale, del "diritto vivente"10.

L'aspetto invece più importante da sottolineare è quello per cui, sulla base della suddetta giurisprudenza costituzionale, appare evidente come una legge possa dare luogo a differenti interpretazioni, tutte astrattamente possibili e come la scelta tra di esse debba essere orientata alla luce della Costituzione, preferendo cioè quella più in linea con il dettato costituzionale. E' immediatamente percepibile, in altri termini, che l'operazione condotta dalla Corte costituzionale attraverso le pronunce interpretative può (o deve) essere operata anche da parte del giudice comune nel decidere le controversie ad esso proposte.

La magistratura pare chiaramente prendere coscienza del significato e del ruolo che la Costituzione viene in tal modo ad assumere per l'attività interpretativa del diritto e degli insegnamenti forniti dalla ricordata giurisprudenza costituzionale. Nel corso del Congresso che l'Associazione nazionale magistrati tiene a Gardone nel 1965 fu infatti approvato un ordine del giorno in cui si respingeva la pretesa di ridurre l'interpretazione "ad una attività puramente formalistica indifferente al contenuto ed all'incidenza concreta della norma nella vita del paese” e, nel delineare la posizione del giudice di fronte alla legge, veniva espressamente indicata la necessità di interpretare la stessa alla luce della Costituzione, seguendo un'interpretazione “adeguatrice” e, solo quando la lettera della legge non lo consentisse, veniva indicata la necessità di proporre alla Corte una questione di legittimità costituzionale. Nonostante ciò quest'ultima soluzione viene in pratica seguita con maggiore frequenza rispetto alla prima, anche perché solo così (attraverso l'autorevole interpretazione fornita dal Giudice costituzionale o la dichiarazione di incostituzionalità della norma impugnata) si è certi di poter superare la diversa interpretazione fatta propria dalla Corte di cassazione.

\footnotetext{
${ }^{9}$ MEZZANOTTE, La Corte costituzionale: esperienze e prospettive, in Attualità e attuazione della Costituzione, Bari, 1979, 160

${ }^{10}$ Per una ricostruzione dei rapporti tra Corte costituzionale e Corte di cassazione e delle vicende che li hanno caratterizzati, v. CAMPANELLI, Incontri e scontri tra Corte suprema e Corte costituzionale in Italia e in Spagna, Torino, 2005, spec. 157 ss.
} 
Si viene cioè a determinare una sorta di alleanza tra la parte più giovane della magistratura e la Corte costituzionale a favore della realizzazione dei principi espressi dalla Costituzione ${ }^{11}$.

A quest'ultimo proposito viene poi presa in considerazione anche l'ipotesi di un'applicazione diretta della Costituzione in tutti quei casi in cui esistono nella legge clausole generali da specificare oppure veri e propri vuoti normativi da riempire, applicando i principi generali dell'ordinamento e quindi in primis i principi costituzionali.

In questi casi si è parlato, a mio avviso impropriamente, di "uso alternativo del diritto", ad indicare un comportamento tendente, in nome dei principi costituzionali e facendo diretta applicazione degli stessi, alla disapplicazione della legge. Come da tempo è stato sottolineato, parrebbe preferibile parlare in proposito di un "uso costituzionale" del diritto, dal momento che il giudice non procede affatto a disapplicare una legge, bensì a riempire i vuoti esistenti, specie in certe materie, e con riferimento ai diritti sociali (si pensi all'ambito dei diritti della persona, al diritto al lavoro, alla salute, solo per fare qualche esempio), derivando la soluzione direttamente dalla Costituzione $^{12}$.

Per quanto concerne invece l'aspetto relativo all'ordinamento giudiziario, sono da segnalare in questo periodo importanti provvedimenti o decisioni che avranno in seguito un ruolo determinante nella realizzazione del modello italiano di ordinamento giudiziario. Mi riferisco alla legge che ha istituito il Consiglio superiore della magistratura - il quale pertanto inizia a funzionare pur se con una serie di limitazioni previste dalla legge del 1958 e poi eliminate dal legislatore o da decisioni di accoglimento della Corte costituzionale - nonché agli interventi legislativi in materia di “carriera” del giudice (quello del 1963, del 1966 e, con riguardo alla nomina a magistrato di cassazione, del 1973).

Di notevole importanza è poi una pronuncia della Corte costituzionale ${ }^{13}$, con la quale essa offre una lettura del principio del giudice naturale precostituito per legge (art. 25, $1^{\circ}$ comma, Cost.), secondo cui esso trova il suo campo di applicazione nelle regole della competenza e non nei rapporti di giurisdizione (divieto di giudici speciali o straordinari). La Corte deriva dal principio in questione una riserva assoluta di legge in materia di competenza del giudice, la quale deve essere indicata preventivamente e direttamente dalla legge, con esclusione di qualsiasi intervento di carattere discrezionale da parte di altri soggetti, sia esterni che interni alla magistratura.

Nei successivi sviluppi - legati alle diverse questioni costituzionalità relative a supposte violazioni della riserva di legge e soprattutto alla riferibilità della garanzia all'organo giudicante astrattamente inteso oppure alle persone fisiche componenti l'organo - il diffondersi, anche a livello normativo e regolamentare, della c.d. cultura del giudice naturale porterà alla creazione del sistema tabellare, il quale ha svolto e

\footnotetext{
${ }^{11}$ In proposito v., volendo, ROMBOLI, L'applicazione della Costituzione da parte del giudice comune, in Ordinamento giudiziario e forense, a cura di Panizza, Pizzorusso e Romboli, Pisa, 2002, 249 ss.

${ }^{12} \mathrm{Cfr}$., tra gli altri, NATOLI, Alcune osservazioni su Costituzione, interpretazione evolutiva e giurisprudenza alternativa, in Legge, giudici, politica. Le esperienze italiana ed inglese a confronto, Milano, 1983, vol. I, $281-282$. ${ }^{13}$ Corte cost. 7 luglio 1962 n. 88, in Giur. cost., 1962, 959, con nota di Micheli.
} 
continua a svolgere una funzione di primissimo piano in ordine alla realizzazione in particolare dell’indipendenza interna del giudice.

3. SEGUE: LA VALORIZZAZIONE ED I LIMITI DELL'ATTIVITÀ INTERPRETATIVA DEL GIUDICE. LA FORMAZIONE DI UN MODELLO ITALIANO DI ORDINAMENTO GIUDIZIARIO

La successiva giurisprudenza costituzionale tende ad una chiara e progressiva valorizzazione dell'attività interpretativa del giudice, sia come conseguenza del ricorso a determinate tecniche decisorie (il giudizio per valori, il necessario bilanciamento, il controllo della ragionevolezza della leggi), sia attraverso affermazioni specificamente rivolte ai poteri interpretativi del giudice con riguardo alla soluzione dell'incidente di costituzionalità.

Così in varie occasioni la Corte costituzionale ha precisato che il giudice, di fronte a più possibili interpretazioni, ha innanzi tutto l'obbligo di scegliere quale di esse intende seguire, escludendo che egli possa sollevare una questione di costituzionalità al solo scopo di richiedere alla Corte la soluzione di un mero dubbio interpretativo, dal momento che ciò rientra nei poteri dell'autorità giudiziaria oppure che un'eccezione di costituzionalità possa essere proposta alla Corte al solo scopo di far prevalere un'interpretazione della legge rispetto ad altra, dovendo essere il giudice a scegliere l'interpretazione conforme alla Costituzione. Un'affermazione in proposito assai importante, più volte ripetuta negli ultimi anni da parte del Giudice costituzionale, è quella secondo cui una legge non può essere denunciata e dichiarata incostituzionale solo perché è possibile darne interpretazioni incostituzionali, ma deve esserlo solamente quando è impossibile darne interpretazioni costituzionalmente conformi ${ }^{14}$.

La tendenza che emerge dalla sopracitata giurisprudenza costituzionale è chiaramente nel senso di sollecitare il giudice a fare uso dei propri poteri interpretativi allo scopo di valutare, preventivamente, se esiste la possibilità di superare i dubbi di costituzionalità attraverso un'interpretazione “adeguatrice”, evitando così di coinvolgere nella soluzione del dubbio la Corte costituzionale, la quale pare recentemente impegnata a valorizzare appunto l'attività interpretativa della legge e della Costituzione (e della prima alla luce della seconda) da parte del giudice, evitando di assumere essa stessa interpretazioni “militanti”, come avveniva con le decisioni interpretative di rigetto della prima maniera.

Nello stesso periodo il nostro modello di ordinamento giudiziario ${ }^{15}$ viene a realizzarsi attraverso la progressiva attuazione dei principi costituzionali, ed in particolare di quelli della autonomia dell’ordine giudiziario e della indipendenza della magistratura. Ciò è dovuto in larga parte all'attività del Consiglio superiore della magistratura, anche grazie alla quale il nostro modello di ordinamento giudiziario trova i suoi punti

\footnotetext{
${ }^{14}$ Sul rapporto tra Corte costituzionale e giudici comuni nell'attività di interpretazione della legge alla luce dei principi costituzionali e l'atteggiamento della prima nel senso di valorizzare la collaborazione ed il diretto coinvolgimento dei secondi nella risoluzione del dubbio di costituzionalità, v. MALFATTI, PANIZZA, ROMBOLI, Giustizia costituzionale, Torino, 2003, 313 ss.

${ }^{15}$ In ordine alla evoluzione ed alla realizzazione di un modello italiano di ordinamento giudiziario, v. PIZZORUSSO, Introduzione, in Ordinamento giudiziario e forense cit., 5 ss.
} 
essenziali nella indipendenza esterna dei giudici - specie nei suoi rapporti con il potere politico - nella indipendenza interna - garantita dalla progressione a ruoli aperti, dal sistema di organizzazione del lavoro secondo il metodo tabellare, dalla applicazione, anche a livello legislativo, del principio di precostituzione del giudice e della logica ad esso sottostante - nonché da un pubblico ministero, garantito al pari del giudice quanto ad indipendenza esterna, con una sempre maggiore valorizzazione del profilo di indipendenza interna e caratterizzato dal principio costituzionale dell'obbligatorietà dell'azione penale.

Gli sviluppi più recenti della giurisprudenza costituzionale in ordine ai poteri interpretativi del giudice comune confermano la tendenza a valorizzare la funzione interpretativa del giudice e la spingono ancora più avanti, tanto da destare di recente, come adesso dirò, una qualche reazione da parte della Corte di cassazione.

Una evidente valorizzazione deriva infatti senza dubbio dalle pronunce della Corte costituzionale che vengono qualificate come "di principio”, le quali possono poi contenere una dichiarazione di incostituzionalità, di infondatezza o di inammissibilità. L'elemento qualificante consiste nel fatto che, attraverso queste pronunce, la Corte non fornisce essa stessa l'interpretazione corretta della disposizione impugnata, né procede ad una diretta manipolazione del testo normativo (come accade attraverso le dichiarazioni di incostituzionalità a carattere additivo, manipolativo o sostitutivo, con cui si procede direttamente a determinare l'effetto di una norma immediatamente applicabile da parte del giudice), ma si limita a fissare appunto un principio, tratto dalla Costituzione, demandando quindi al giudice (ed in alcuni casi anche al legislatore) il compito di trovare la soluzione interpretativa più idonea per il caso da decidere alla luce ed in applicazione del principio enunciato dalla Corte. Ciò nella considerazione che, in alcune situazioni, la conformazione della legislazione ai principi costituzionali sia meglio perseguibile con riferimento al caso concreto che non attraverso una pronuncia demolitoria della Corte costituzionale (si pensi ai casi della spirale delle condanne per gli obiettori di coscienza, del disconoscimento di paternità in caso di inseminazione artificiale eterologa, della differenza di età tra adottante ed adottato ai fini dell'adozione, solo per fare alcuni recenti esempi).

La richiesta, da parte della Corte, al giudice di seguire, quando possibile, un'interpretazione della disposizione impugnata che valga a salvare la stessa dalla dichiarazione di incostituzionalità, dapprima presentata come un invito, una richiesta al giudice $a$ quo, si è in questi ultimi anni trasformata in un obbligo per quanti intendano proporre eccezioni di costituzionalità. Accanto infatti alle tradizionali condizioni, poste dalla legge, della "rilevanza" e della "non manifesta infondatezza" che l'autorità giudiziaria deve necessariamente valutare prima di rimettere una questione alla Corte, questa ha adesso aggiunto, in via giurisprudenziale, una terza condizione rappresentata dall'aver il giudice sperimentato previamente la possibilità di una interpretazione "adeguatrice" o "conforme". La questione di costituzionalità infatti viene attualmente dichiarata inammissibile (o più spesso manifestamente inammissibile) qualora esista, in giurisprudenza, una diversa lettura della disposizione impugnata che superi i dubbi di costituzionalità e comunque nel caso in cui il giudice 
rimettente non dimostri di aver sperimentato la possibilità di giungere ad una interpretazione "conforme" a Costituzione ${ }^{16}$.

In una recente occasione - relativa ad una questione di costituzionalità circa la mancata previsione del rilascio del permesso di soggiorno, al compimento della maggiore età, per i minori stranieri sottoposti a tutela, quando ciò era invece previsto per i minori dati in affidamento - la Corte costituzionale ha rilevato come l'interpretazione letterale della disposizione impugnata poneva la stessa in sicuro contrasto con il principio personalista della nostra Costituzione ed ha suggerito al giudice, attraverso una pronuncia di infondatezza, una integrazione della disposizione in via analogica, sulla base della comparazione fra i presupposti e le caratteristiche del rapporto di tutela del minore e del rapporto di affidamento, concludendo che, nonostante la diversa previsione normativa, la disposizione impugnata doveva ritenersi riferita anche ai minori stranieri sottoposti a tutela ${ }^{17}$.

Una ancora più recente pronuncia della Corte di cassazione ${ }^{18}$, ha posto in evidenza il problema e fatto parlare, a mio avviso impropriamente, di una "riapertura della guerra tra le due corti”.

Volendo sintetizzare al massimo la questione, ai limitati fini del discorso che sto svolgendo, la Corte costituzionale aveva risolto una questione di costituzionalità attraverso una pronuncia interpretativa di rigetto con la quale indicava al giudice l'interpretazione "conforme” da seguire. La Corte di cassazione, pur non ponendo in discussione la possibilità per il Giudice costituzionale di fare pronunce interpretative, faceva notare come nella specie fosse impossibile al giudice comune seguire l'interpretazione suggerita dalla Corte costituzionale, in quanto essa si poneva in palese contrasto con la lettera della legge, la quale poteva essere superata solamente attraverso una pronuncia di incostituzionalità. A fronte della nuova questione di costituzionalità, per tali motivi riproposta dalla Corte di cassazione, il Giudice

${ }^{16}$ Circa l'obbligo, per il giudice, di dimostrare, pena la dichiarazione di inammissibilità della questione di costituzionalità, di aver sperimentato la possibilità di superare il dubbio di costituzionalità attraverso un'interpretazione "adeguatrice", con riguardo alla più recente giurisprudenza costituzionale, v. ROMBOLI, Il giudizio di costituzionalità delle leggi in via incidentale, in Aggiornamenti in tema di processo costituzionale (2002-2004), Torino, 2005, 76 ss.

${ }^{17}$ Corte cost. 5 giugno 2003, n. 198, Giur. cost., 2003, 1520. Il giudice a quo rilevava come la disposizione impugnata prevede la possibilità che sia rilasciato, in certe condizioni, il permesso di soggiorno al minore straniero in condizione di affidamento e non anche per quelli sottoposti a tutela, osservando altresì come, stante la chiara ed inequivoca lettera della legge, non era ammissibile un'interpretazione estensiva tale da ritenere la stessa previsione valida anche per la differente situazione della tutela. Per questo l'autorità giudiziaria domandava alla Corte una pronuncia di incostituzionalità della disposizione impugnata, per la parte in cui non riteneva applicabile quanto in essa previsto anche alla situazione dei minori sottoposti a tutela.

La Corte ha invece affermato che lo stesso risultato poteva essere raggiunto, se non attraverso un'interpretazione estensiva, comunque attraverso un'integrazione in via analogica, sulla base della comparazione fra i presupposti e le caratteristiche del rapporto di tutela del minore e del rapporto di affidamento, entrambi finalizzati ad assicurare la cura del minore, nonché sulla base della sostanziale eguaglianza delle situazioni di fatto nelle quali si trovano i minori stranieri in affidamento o sottoposti a tutela.

${ }^{18}$ Cass. 31 marzo 2004, Pezzella, Foro it., 2004, II, 337. Per una ricostruzione della vicenda v. SORRENTI, “...la sventurata rispose" (ovvero: interpretazione plausibile versus interpretazione verfassungskonforme della legge nella sent. n. 23016/2004 della Corte di cassazione, S.U. penali), in Scritti dei dottorandi in onore di Alessandro Pizzorusso, Torino, 2005, 365 ss. 
costituzionale ha dichiarato manifestamente inammissibile l'eccezione, limitandosi a ribadire la propria interpretazione "conforme" della disposizione impugnata, senza prendere espressamente in considerazione le ragioni addotte per la riproposizione dell'eccezione di costituzionalità dalla Cassazione. Quest'ultima ha di conseguenza ritenuto di non poter seguire l'interpretazione suggerita dalla Corte costituzionale, non essendo consentito al giudice di procedere direttamente ad una disapplicazione della legge e di non poter neppure risollevare l'eccezione di costituzionalità, data la risposta ricevuta dalla Corte costituzionale ed ha pertanto fatto applicazione della disposizione censurata, nella interpretazione ritenuta dallo stesso giudice di dubbia costituzionalità.

A fronte della medesima eccezione, stavolta sollevata da un giudice di merito, la Corte costituzionale non ha potuto che prendere atto della posizione assunta in proposito dalla cassazione e del fatto che poteva ritenersi formato un diritto vivente incompatibile con l'interpretazione suggerita dalla stessa Corte e quindi ha provveduto a dichiarare l'incostituzionalità della norma impugnata ${ }^{19}$.

Il problema che emerge dalla vicenda è quello relativo ai limiti entro cui, nel nostro ordinamento delle fonti, è consentito al giudice di superare i dubbi di costituzionalità attraverso l'interpretazione (o anche l'analogia) “adeguatrice” e quando invece, non potendo esso disapplicare la legge supposta in contrasto con la Costituzione, l'intervento demolitorio della Corte costituzionale si ponga come assolutamente indispensabile.

L'atteggiamento in proposito tenuto dalla Corte di cassazione potremmo sintetizzarlo nel senso che essa, mentre - superati i problemi che si erano posti alla base della c.d. guerra delle due corti (legalità-costituzionalità) - accetta ormai pacificamente che sia la Corte costituzionale a fornire l'interpretazione della legge costituzionalmente corretta e che il giudice debba seguire la stessa ${ }^{20}$ - non accetta quelle interpretazioni che vadano chiaramente contro la lettera della legge, nel senso che il giudice di legittimità non ritiene vincolanti quelle interpretazioni, contenute nella motivazione della sentenza costituzionale, che assumono natura "manipolativa" (additiva, sostitutiva o comunque innovativa) del testo legislativo, riconoscendo che un tale effetto possa discendere solo da dichiarazioni demolitorie o comunque aventi un dispositivo di incostituzionalità.

Nel valorizzare il ruolo del giudice la Corte costituzionale pare in qualche misura spingere verso una progressiva, maggiore espansione della funzione dello stesso di "giudice dei diritti” - rispetto a quella di "giudice della legalità" ${ }^{21}$ - venendo, con qualche vaga somiglianza all'attività propria della Corte di Lussemburgo, a dare essa la corretta interpretazione della Costituzione e della legge alla luce della prima o fissando i principi e lasciando poi al giudice di risolvere il caso specifico secondo quella interpretazione o in applicazione di quei principi, anche quando ciò comporti

${ }^{19}$ Corte cost. 22 luglio 205, n. 299, G.U., $1^{\circ}$ s.s., 27 luglio 2005, n. 30.

${ }^{20}$ Cass. 16 dicembre 1998, Scorzelli, Giur. cost., 1999, 1402, con nota di LAMARQUE, Le sezioni unite penali della Cassazione "si adeguano" ... all'interpretazione adeguatrice della Corte costituzionale.

${ }^{21}$ In ordine alla evoluzione del rapporto fra giudice e legislatore, nel senso della accentuazione del ruolo di "giudice dei diritti” rispetto a quello di "giudice della legalità", v. F.G.PIZZETTI, Il giudice nell'ordinamento complesso, Milano, 2003, 243 ss. 
una evidente forzatura (che pare, in certi casi, quasi avvicinarsi ad una disapplicazione) della disposizione impugnata.

4. IL RUOLO DEL GIUDICE A SEGUITO DELLA REALIZZAZIONE DELL'ORDINAMENTO COMUNITARIO: LA PREGIUDIZIALITÀ COMUNITARIA ED IL POTERE DI DISAPPLICAZIONE DELLA LEGGE NAZIONALE

Il secondo momento da sottolineare per l'incidenza sul ruolo del giudice, nell'evoluzione del sistema delle fonti del diritto, è rappresentato, come dicevo, dalla realizzazione dell'ordinamento comunitario, a proposito del quale si parla della creazione di un nuovo diritto comune europeo, fondato finora, e nel prossimo futuro, in maniera determinante dal confronto tra le decisioni giurisprudenziali che, a diverso livello, assumeranno gli organi giurisdizionali nazionali e sopranazionali. Un esplicito riconoscimento del valore della giurisprudenza per la realizzazione dell'ordinamento comunitario è contenuto nel preambolo della Carta di Nizza, ora incorporata nella c.d. Costituzione europea, dove si afferma che la Carta riafferma "i diritti riconosciuti dalla giurisprudenza della Corte di giustizia delle Comunità europee e da quella della Corte europea dei diritti dell'uomo”.

Volendo richiamare i punti di arrivo di quello che è detto il "cammino comunitario", per quanto concerne in specifico i riflessi sul sistema delle fonti e quindi sul ruolo del giudice, possiamo sottolineare, da un lato, l'affermazione del primato del diritto comunitario su quello nazionale - essendo all'evidenza uscita ormai definitivamente sconfitta la teoria dualista, ancora a parole riaffermata dalla nostra Corte costituzionale, a vantaggio della teoria monista da sempre sostenuta dalla Corte europea - e, dall'altro, il conseguente potere-dovere del giudice di sottoporre, in caso di dubbio ed in via pregiudiziale, alla Corte di Lussemburgo la questione relativa all'interpretazione del diritto comunitario, nonché l’obbligo di disapplicazione (o non applicazione) del diritto nazionale, qualora lo stesso sia ritenuto in contrasto con il diritto comunitario.

L'importanza ed il ruolo centrale che viene ad assumere il giudice nazionale deriva dal fatto che la Corte di giustizia, al fine di rendere efficaci i vincoli derivanti per gli stati membri dal diritto comunitario, ha pensato di appoggiare il compito di controllare, e in parte di sanzionare, gli stati ai loro giudici nazionali, attraverso appunto gli strumenti della pregiudiziale comunitaria e del potere-dovere di disapplicazione. Il ricorso da parte del giudice alla Corte europea per la interpretazione pregiudiziale del diritto comunitario è poi venuto ad assumere un'efficacia decisamente maggiore a seguito del riconoscimento, da parte della Corte costituzionale, della efficacia di fonte del diritto anche alle sentenze interpretative della Corte di Lussemburgo ${ }^{22}$, come ribadito di recente dalla giurisprudenza costituzionale che ha restituito gli atti al giudice a quo per un riesame della rilevanza a seguito di "ius superveniens", dove lo "ius" era appunto rappresentato da una sentenza interpretativa della Corte di giustizia ${ }^{23}$.

\footnotetext{
${ }^{22} \mathrm{Si}$ veda in proposito MARTINICO, Le sentenze interpretative della Corte di giustizia come forme di produzione normativa, in Riv. dir. cost., 2004, 249 ss.

${ }^{23}$ Corte cost., ord. 14 marzo 2003, n. 62 e ord. 20 aprile 2004 n. 125, Foro it., 2004, I, 2299, le quali si fondano sull'affermazione secondo cui i principi enunciati nelle decisioni della Corte di giustizia si inseriscono direttamente nell'ordinamento interno ed hanno quindi valore di fonte del diritto e di ius superveniens.
} 
Attraverso la possibilità di disapplicazione della fonte nazionale, in quanto contrastante con il diritto comunitario, il giudice comune viene così ad esercitare una sorta di controllo diffuso di "comunitarietà” nei confronti della legge nazionale, che segue spesso al rinvio pregiudiziale. Quest'ultimo infatti viene ad assumere caratteri, per certi versi simili a quelli della pregiudiziale di costituzionalità davanti alla Corte costituzionale, dal momento che, com'è stato opportunamente notato ${ }^{24}$, si viene, di fatto, ad esercitare un controllo sulla conformità della legge nazionale al diritto comunitario allorché la Corte di giustizia, come spesso accade, non si limita a rispondere al quesito posto dal giudice, ma si spinge oltre dando esplicitamente un giudizio in ordine ad una determinata disciplina ("la direttiva X osta ad una disciplina nazionale che preveda...” oppure "l'art. Y del TCE deve interpretarsi nel senso che osta ad una regola quale quella dell'art. Z della legge nazionale XY”).

Con riguardo all'atteggiamento tenuto in proposito da parte della magistratura, si è venuta, mutatis mutandis, a riprodurre in qualche misura quella situazione già verificatasi con riguardo alle questioni pregiudiziali di costituzionalità, ossia una maggiore sensibilità alla pregiudiziale comunitaria da parte dei magistrati più giovani rispetto a quelli più anziani, dando luogo al fenomeno per cui ad esempio, nel corso del 2003, la Corte di cassazione non ha sollevato neppure una questione pregiudiziale ed il Consiglio di Stato solamente tre, pur tenendo conto del fatto che, mentre gli altri giudici hanno la facoltà di ricorrere alla Corte di giustizia, essa diviene un obbligo per i giudici di ultima istanza.

Il giudice nazionale viene pertanto a trovarsi nella condizione di dover seguire l'interpretazione conforme, non solamente con riguardo alla Costituzione, ma pure con riferimento al diritto comunitario (così come, in varie occasioni, sottolineato dalla Corte di giustizia) e, allorché non ritenga possibile fare ciò direttamente, è tenuto ad investire, attraverso la richiesta pregiudiziale, la Corte costituzionale oppure la Corte di giustizia. In certe ipotesi può darsi che le due vengano a porsi entrambe, contemporaneamente, con riferimento ad uno stesso caso, determinandosi il fenomeno della c.d. doppia pregiudizialità ${ }^{25}$, con la necessità per il giudice di stabilire in quale rapporto stiano tra di loro l'una e l'altra e quale quindi debba essere esercitata per prima. In proposito la Corte costituzionale ha ritenuto che la pregiudiziale comunitaria debba precedere quella costituzionale ed infatti, anche di recente, ha rinviato a nuovo ruolo (in attesa della decisione della Corte di giustizia) la pronuncia in ordine ad una questione di legittimità costituzionale sollevata da un giudice in via incidentale, sulla base dell'informazione, comunicata in udienza dall'Avvocatura dello Stato, che la medesima questione era stata sollevata, da altri giudici, alla Corte di Lussemburgo, per un'interpretazione ai sensi del TCE ${ }^{26}$.

${ }^{24}$ Cfr. CALVANO, La Corte di giustizia e la Costituzione europea, Roma, 2004, 240 ss.; GIOVANNETTI, Riflessioni sul controllo di legalità comunitaria tra Corte costituzionale, giudici comuni e Corte di giustizia, in Scritti dei dottorandi cit., 421 ss.

${ }^{25}$ Sulla quale v., di recente, SORRENTINO, E' veramente inammissibile il "doppio rinvio"?, in Giur. cost., 2002, 783 ss.; CELOTTO, Ancora un'occasione perduta per mettere chiarezza sulle interferenze tra giudizio di costituzionalità e giudizio di "comunitarietà", ivi, 2004, 1732 ss.

${ }^{26}$ Corte cost., ord. $1^{\circ}$ giugno 2004, n. 165, Foro it., 2004, I, 2299. Di fronte ad un giudice che aveva esercitato entrambe le pregiudizialità contemporaneamente, la Corte ha risposto dichiarando manifestamente 
Attraverso il raccordo con la Corte di giustizia è pertanto consentito al giudice comune di superare, dandosi certe condizioni, anche l'interpretazione fornita dalla Corte costituzionale e soprattutto quella del legislatore nazionale. Esemplare in proposito il recente caso in materia ambientale, relativo alla nozione di "rifiuto" di cui all'art. 6, $1^{\circ}$ comma, lett. a), d. lgs. 5 febbraio 1997 n. 22, sul quale si era creata una giurisprudenza dei giudici comuni ormai consolidata, fondata principalmente sulla giurisprudenza della Corte di giustizia. Il governo italiano ha ritenuto quella interpretazione particolarmente restrittiva e tale da poter pregiudicare la competitività sul mercato di vari settori della nostra industria e, di conseguenza, ha approvato un decreto-legge (8 luglio $2002 \mathrm{n}$. 138, convertito in legge 8 agosto 2002 n. 178), con cui ha fornito l'interpretazione autentica della nozione di "rifiuto", in palese contrasto con la giurisprudenza comunitaria. Alcuni giudici nazionali hanno in seguito proposto questione pregiudiziale, chiedendo alla Corte di giustizia di pronunciarsi in merito alla compatibilità con il diritto comunitario della nozione di "rifiuto" desumibile dalla legge di interpretazione autentica ed essa si è espressa in senso negativo, confermando la propria precedente giurisprudenza in materia $\left({ }^{27}\right)$ e consentendo al giudice di disapplicare la normativa nazionale.

Il ruolo pertanto che viene richiesto al giudice nazionale, a seguito della realizzazione dell'ordinamento comunitario, è pertanto veramente centrale e complesso, egli infatti, come ha sostenuto il presidente della Corte di giustizia Rodriguez Iglesias ${ }^{28}$, ha due cappelli, ma li porta contemporaneamente e non uno dopo l'altro.

Con l'ordinamento comunitario le funzioni del giudice vengono quindi ad ampliarsi ed arricchirsi di nuovi strumenti ed istituti, che producono inevitabilmente un ampliamento del proprio potere interpretativo, che lo conduce, come detto, alla possibilità anche di non dare applicazione ad una legge dello stato. Il giudice infatti viene a rivestire il ruolo di giudice della legalità dell'ordinamento, anche se rispetto ad esso viene ad avere sempre maggiore rilievo quello di giudice dei diritti; il giudice è il portiere (l'unico portiere potremmo aggiungere) della Corte costituzionale, ma deve evitare di aprire la porta quando il dubbio di costituzionalità può essere superato facendo ricorso ai propri poteri interpretativi (c.d. interpretazione conforme) e può, ricorrendone le condizioni, risolvere il caso applicando direttamente la Costituzione; il giudice deve pure perseguire un'interpretazione adeguatrice della normativa nazionale al diritto comunitario e, quando necessario, proporre la questione interpretativa alla Corte di giustizia di Lussemburgo, nonché, anche sulla base della risposta di quest'ultima, procedere alla diretta disapplicazione (o non applicazione) della legge nazionale ritenuta in contrasto con il diritto comunitario.

inammissibile la questione di costituzionalità, per manifesta contraddittorietà della stessa, in quanto la pregiudiziale comunitaria tende ad accertare se la norma censurata sia compatibile con l'ordinamento comunitario, e quindi applicabile nell'ordinamento italiano, mentre la questione di costituzionalità presuppone che la norma, di cui il giudice stesso ha sollecitato l'interpretazione della Corte di giustizia, sia applicabile (ord. 21 marzo 2002, n. 85, Foro it., 2002, I, 1603).

${ }^{27}$ Corte giust. Cee, sez. II, 11 novembre 2004, causa C457/02, Foro it., 2005, IV, 16, con nota di AMENDOLA, "Rifiuto": non era autentica l'interpretazione italiana e di PAONE, La nozione di rifiuto tra diritto comunitario e diritto penale.

${ }^{28}$ RODRIGUEZ IGLESIAS, Intervento all'Incontro di studio tra la Corte costituzionale e la Corte di giustizia delle comunità europee, Roma, Palazzo della Consulta, 4-5 aprile 2002, Roma, 2003. 
In questa molteplicità di funzioni e quindi anche di ruoli che vengono richiesti al giudice e, specie di fronte al diffondersi di quella che è stata definita la cultura della disapplicazione, può anche capitare che il giudice si confonda e pronunci provvedimenti, giustamente criticati da una larga parte della dottrina, in cui ad esempio la disapplicazione della legge viene fatta discendere direttamente dal richiamo alle disposizioni della Carta di Nizza, ancora prive di valore giuridico ${ }^{29}$.

Il comprensibile disorientamento del giudice a fronte dei diversi strumenti disponibili pare risultare anche da una recente pronuncia giurisdizionale, impugnata dalla regione attraverso lo strumento del conflitto tra enti, la quale denunciava il fatto che il giudice, dopo aver rilevato un vizio di costituzionalità della legge regionale, anziché sollevare la relativa questione di costituzionalità, aveva illegittimamente proceduto alla disapplicazione della legge medesima ${ }^{30}$.

5. LA PRODUZIONE DEL DIRITTO DA PARTE DEL LEGISLATORE E DA PARTE DEL GIUDICE: FORME, MODALITÀ E LEGITTIMAZIONE.

A fronte del ruolo che il giudice è chiamato oggi ad assolvere e che ho cercato sommariamente di riassumere, nei suoi elementi a mio avviso più rilevanti, il problema della natura creativa o meno dell'attività interpretativa o della ricerca e ricostruzione della volontà del legislatore (o dei legislatori, come sarebbe più corretto dire) potrebbe essere risolto attraverso due sole parole, quelle usate in proposito venti anni fa da Mauro Cappelletti: "verità banale”. Egli infatti nella premessa al suo volume “Giudici legislatori?”, osservava come lo scopo dello scritto non era certamente quello di "dimostrare la verità banale, anche se infinite volte in ogni epoca e con tanta inesauribile perseveranza negata o nascosta, della creatività della giurisprudenza”31.

Affermare la natura creativa della giurisprudenza significa ammettere - senza poter affrontare il delicato tema del valore del precedente giudiziario come fonte del diritto, anche quando ad esso non viene formalmente riconosciuta forza vincolante che anche i giudici, nei limiti e nelle forme fissati dall'ordinamento, concorrono alla determinazione ed alla produzione del diritto. Ciò non vuol dire affatto ovviamente porre questi ultimi sullo stesso piano del legislatore, ma che il principio di soggezione del giudice alla legge significa innanzi tutto, come da tempo affermato e dimostrato da Pizzorusso ${ }^{32}$, soggezione al diritto e che il rapporto tra legislatore e giudici non è, come già sostenuto da Carlo Esposito ${ }^{33}$ e da Salvatore Satta ${ }^{34}$, quello di una "assurda gerarchia”, ponendosi i due soggetti in una posizione di assoluta parità, ognuno

${ }^{29}$ Cfr. CARTABIA, CELOTTO, La giustizia costituzionale in Italia dopo la Carta di Nizza, in Giur. cost., 2002, 4477 ss.

${ }^{30}$ La Corte costituzionale (sent. 28 aprile 2004, n. 129, Foro it., 2004, I, 1985) ha accolto il ricorso della regione e, quindi, annullato il provvedimento giurisdizionale impugnato, trascurando del tutto il fatto che, a ben vedere, il giudice aveva non solamente rilevato il possibile vizio di costituzionalità, ma anche il suo contrasto con una direttiva comunitaria, ritenuta direttamente efficace nel nostro ordinamento, fondando su quest'ultimo rilievo la decisione di disapplicare la legge regionale.

${ }^{31}$ CAPPELLETTI, Giudici legislatori? cit., I.

${ }^{32}$ PIZZORUSSO, Intervento al Convegno di Senigaglia 9-11 novembre 1979, in Giust. e Cost., 1980, 3-4, 183 ss e Intervento, in I poteri del giudice civile di fronte alla legge, Rimini, 1985, 219 ss.

${ }^{33}$ SATTA, Norma, diritto, giurisdizione, in Studi in memoria di Carlo Esposito, Padova, 1973, III.

${ }^{34}$ ESPOSITO, La validità delle leggi, Milano, 1934. 
certamente secondo il proprio ruolo, nello svolgimento e nella determinazione dell'ordinamento giuridico.

Come scrive Gustavo Zagrebelsky, chiudendo il suo notissimo scritto sul diritto mite, il legislatore deve rassegnarsi a vedere le proprie leggi trattate come "parti" del diritto, non come "tutto il diritto", né i giudici possono ritenersi i padroni del diritto nello stesso senso in cui il legislatore lo era nel secolo scorso, essendo essi più propriamente i garanti della complessità strutturale del diritto nello stato costituzionale, cioè della necessaria, mite coesistenza di legge, diritti e giustizia; "il diritto non è oggetto in proprietà di uno ma deve essere oggetto delle cure di tanti”35.

La produzione del diritto ad opera del legislatore e del giudice avviene certamente con forme e modalità differenti ed entrambi i soggetti operano nell'ordinamento secondo differenti forme di responsabilità e di legittimazione democratica. Come scrive recentemente Alessandro Pizzorusso, l'atto legislativo è rivolto direttamente a produrre diritto ed impone una regola per il futuro, mentre l'atto giurisdizionale è rivolto a risolvere una controversia sulla base di norme previgenti, con l'effetto solo indiretto di produzione normativa e, mentre il primo è fondato sulla volontà politica, il secondo trova il suo fondamento nella forza della ragione ed è qualificabile come fonte culturale ${ }^{36}$.

La legittimazione dell'attività di produzione del diritto da parte del legislatore si ricollega al rapporto di rappresentanza diretta del corpo elettorale, al quale esso risponderà delle proprie scelte e pertanto le modalità dell'intervento sono tali che spetterà solo ad esso stabilire se farlo, quando farlo e con quale specifico contenuto. $\mathrm{Al}$ legislatore è consentito anche, attraverso l'esercizio del proprio potere normativo, di contrastare eventuali interpretazioni, ritenute non corrette, date alle proprie leggi, attraverso l'approvazione di leggi di interpretazione autentica, le quali possono prevedere anche la loro applicazione per i procedimenti in corso (leggi in varie occasioni ritenute dalla Corte costituzionale non in contrasto con la Costituzione) ed a lui rimane altresì l'ultima parola anche nei riguardi di dichiarazioni di incostituzionalità delle proprie leggi, avendo il potere di revisione costituzionale, seppure è auspicabile che tale potere venga usato solo rarissimamente e per situazioni assolutamente eccezionali ${ }^{37}$.

Diversa è invece da ritenere la fonte di legittimazione dell'attività normativa, nel senso detto, del giudice, il quale incontra innanzi tutto una serie di vincoli e di limiti, a partire ovviamente dalla lettera della legge, dalle regole dell'interpretazione comunemente accettate, nonché, con la dovuta cautela, da quella che Ross chiama la "tradizione culturale”, vale a dire i fattori morali, economici, politici e culturali propri di un paese, nel momento in cui il giudice è chiamato a giudicare.

A ciò deve aggiungersi quella che è stata da alcuni chiamata la "processualità" dell'agire del giudice ${ }^{38}$, che lo distingue nettamente dall'azione del legislatore. Intendo

\footnotetext{
${ }^{35}$ ZAGREBELSKY, Il diritto mite cit., 213.

${ }^{36}$ PIZZORUSSO, Principio democratico e principio di legalità, in Questione giustizia, 2003, n. 2, 340 ss.

${ }^{37}$ Sui rapporti tra parlamento e Corte costituzionale in ordine alla riproduzione di una legge dichiarata incostituzionale attraverso una legge costituzionale o di revisione costituzionale, v. MALFATTI, PANIZZA, ROMBOLI, Giustizia costituzionale, cit., 306 ss.

${ }^{38}$ Così CAPPELLETTI, Giudici legislatori cit., 126.
} 
riferirmi al principio per cui il giudice deve agire solo dietro richiesta degli interessati (ne procedat iudex ex officio), al rispetto delle garanzie di imparzialità, alla tutela del contraddittorio, alla necessaria motivazione delle proprie decisioni. In tutto questo può ritenersi che si sostanzia oggi il significato del principio della soggezione del giudice alla legge (rectius al diritto), da cui abbiamo preso le mosse ${ }^{39}$.

6. IL RUOLO ATTUALE DEL GIUDICE E LA LEGGE N. 150 DEL 2005 DI RIFORMA DELL'ORDINAMENTO GIUDIZIARIO.

Come ho cercato di esporre, la trasformazione e l'evoluzione del ruolo del giudice a seguito della modificazione del sistema delle fonti è, in certa misura, andata di pari passo ad un determinato assetto della struttura organizzativa, vale a dire del modello di ordinamento giudiziario, essendo inevitabilmente l'uno strettamente collegato con l'altro.

Gustavo Zagrebelsky sottolinea giustamente la necessità di ridurre "la distanza che separa la straordinaria profondità e creatività della funzione giudiziaria odierna e l'organizzazione ad essa preposta, originariamente pensata per un corpo di pubblici dipendenti il cui carattere maggiormente apprezzato era l'apatia sociale e l'abitudine di nascondere le proprie decisioni dietro un burocratico 'ita lex",40.

Ci possiamo pertanto, in conclusione, domandare se la legge sull'ordinamento giudiziario, approvata recentemente dal parlamento, si ponga in questa ottica, ossia, in altri termini, risponda al ruolo che l'ordinamento attuale assegna al giudice.

Premetto che il mio giudizio è negativo ed è fondato non tanto su questo o quello aspetto specifico della legge, quanto sullo spirito generale che la anima e che traspare facilmente dall'intera impostazione della legge.

Tralasciando ogni considerazione relativa al particolare momento in cui la legge è stata approvata, segnato da un forte contrasto tra la maggioranza politica e l'ordine giudiziario e tale da far dubitare dell'opportunità di procedere alla approvazione di una legge sull'ordinamento giudiziario, dare oggi attuazione alla VII disposizione transitoria della Costituzione non può significare "riscrivere" la legge sull'ordinamento giudiziario, così come avrebbe potuto (o dovuto) fare il legislatore ordinario nel 1948, bensì "razionalizzare" i risultati che si sono venuti realizzando in questi anni, in applicazione dei principi costituzionali.

Questo in considerazione del fatto che, se è vero che la prevista legge generale di riforma dell'ordinamento giudiziario non è mai stata approvata, è altrettanto vero che la situazione normativa dell'ordinamento giudiziario non è certamente rimasta quella esistente nel 1948. Al contrario, attraverso interventi legislativi parziali, la giurisprudenza della Corte costituzionale, la c.d. attività paranormativa del Csm, l'attività interpretativa dei giudici comuni, si è venuto realizzando, come ho detto, un modello italiano di ordinamento giudiziario, in conformità alla Costituzione.

\footnotetext{
${ }^{39}$ Si vedano in proposito le osservazioni di BIN, Lo stato di diritto, Bologna, 2004, spec. 55 ss. e 115 ss.

${ }^{40}$ ZAGREBELSKY, Il diritto mite cit., 206, il quale ricorda, tra le misure di organizzazione la cui disciplina incide sul ruolo del giudice: le forme di reclutamento, la responsabilità, la valutazione delle loro attitudini, la struttura degli organi preposti alla loro carriera, il modo di organizzare il loro lavoro.
} 
La legge 150/2005 ${ }^{41}$ introduce invece evidenti elementi di gerarchizzazione all'interno della magistratura - cui pare, in qualche misura, collegarsi anche l'introduzione del discusso colloquio di idoneità psico-attitudinale all'esercizio della funzione del magistrato e come mostra inequivocabilmente la nuova disciplina dell'organizzazione interna del pubblico ministero - e tende abbastanza chiaramente ad ottenere un riposizionamento del potere giudiziario nei riguardi degli altri poteri dello stato e di quello esecutivo in particolare, mostrando l'intento di arrestare il processo evolutivo, invertendone l'ordine e tentando di modificare quel modello di ordinamento giudiziario che si è venuto pian piano a realizzare e che ha dato sicurezza e garanzie alla magistratura, consentendole di svolgere la propria delicata funzione in maniera indipendente da pressioni e influenze provenienti dal potere politico ${ }^{42}$.

Senza voler svolgere, in questa sede, un'analisi specifica della legge in questione, vorrei solo limitarmi ad evidenziarne alcuni limitati, ma significativi, aspetti, oltre quelli appena ricordati.

La professionalità del giudice rappresenta, come è stato scritto da Pizzorusso ${ }^{43}$ e da Proto Pisani ${ }^{44}$, una condizione della legittimazione democratica dell'attività giurisdizionale e pertanto essa deve sussistere, come ho avuto modo di notare in altra occasione $^{45}$, “a livello diffuso", dal momento che, a differenza di quanto avviene per i difensori o per altri professionisti, il cittadino non ha la possibilità di scegliersi il giudice competente (possibilità anzi che è espressamente preclusa dal principio costituzionale della precostituzione del giudice per legge) ed ha quindi il diritto che lo stato lo assicuri della professionalità del giudice, quale che sia il magistrato che andrà a giudicarlo. Il controllo della professionalità rappresenta pertanto un momento di estrema delicatezza ed importanza ed il ripristino del sistema dei concorsi operato dalla legge n. 150 del 2005, se sembra destinato, da un lato, a ripetere tutti i difetti che lo stesso già aveva mostrato (conformismo, ritardo nei processi, ricerca di collocazioni idonee o di "nicchie” per preparare il concorso) pare, dall'altro, non aderente al ruolo riconosciuto al giudice dall'attuale ordinamento, in quanto procede ad una selezione dei magistrati fondata principalmente sulla verifica di un'astratta preparazione tecnica (così come avveniva in base al vecchio ordinamento giudiziario), in larga misura simile a quella prevista per l'accesso in magistratura.

Certamente utile allo scopo di favorire la formazione di una maggiore professionalità dei magistrati deve ritenersi l'istituzione di una Scuola della magistratura, la quale

\footnotetext{
${ }^{41}$ Per un primo commento alla l. n. 150 del 2005 v. il fascicolo di Guida al diritto, n. 32 del 13 agosto 2005; AA.VV., La legge di riforma dell'ordinamento giudiziario cit.

${ }^{42}$ Rileva PIZZORUSSO (Considerazioni generali, in AA.VV., La legge di riforma cit.) come, data la rigidità della Costituzione, il radicale contrasto della l. n. 150 con i principi contenuti nella stessa, non solo toglie ogni senso all'affermazione secondo cui tale legge costituirebbe attuazione della VII disp. trans. della Costituzione, ma ne determina anzi la totale ed irrimediabile incostituzionalità proprio per violazione di tale disposizione (oltre che di molte altre contenute nella prima e nella seconda parte della stessa), al di là di specifici motivi di incostituzionalità di molte delle disposizioni particolari che sono in essa contenute. ${ }^{43}$ PIZZORUSSO, Principio democratico e principio di legalità cit., 345 ss.

${ }^{44}$ PROTO PISANI, Controriforma dell'ordinamento giudiziario: ultimo atto?, in Foro it., 2004, 109 ss.

${ }^{45}$ ROMBOLI, La professione del magistrato tra legislazione attuale e possibili riforme, in I magistrati e la sfida della professionalità, Milano, 2003, 9 ss.
} 
pare però, nella legge in questione, essere prevista principalmente con lo scopo di sottrarre competenze al Consiglio superiore della magistratura ${ }^{46}$, in linea con il più generale disegno di determinare un ridimensionamento di tale organo.

Anche la riorganizzazione della Corte di cassazione ed una riflessione sul ruolo che si intende ad essa assegnare (come corte suprema o come terzo grado di giudizio) è da tempo al centro di dibattiti sia in dottrina che nella magistratura, ma la principale preoccupazione in proposito del legislatore pare essere quella, non tanto di migliorare l'attività nomofilattica della stessa o più in genere l'efficienza del servizio da questa fornito, quanto la produzione di nuovi assetti di potere all'interno della magistratura, attribuendo alla Cassazione una posizione dominante, di vertice, come accadeva al momento dell'entrata in vigore della Costituzione e prima che la Corte costituzionale iniziasse a funzionare.

Molto si è discusso in ordine alle fattispecie di responsabilità disciplinare previste dalla legge, sia per alcune ipotesi poi fortunatamente eliminate (tra cui quella che prevedeva il divieto di interpretazione creativa), sia per l'estrema importanza che la disciplina della stessa assume, rappresentando, come è stato sottolineato, il punto di confluenza tra il principio democratico e quello dell'indipendenza della magistratura ${ }^{47}$.

Senza entrare nel merito delle singole fattispecie previste dalla legge, vorrei solo rilevare come da alcune di queste traspare chiaramente come esse paiono pensate come strumento di intimidazione e come tentativo di condizionare il potere interpretativo del giudice (ad esempio la previsione, come illecito disciplinare, dell'adozione di provvedimenti che "costituiscano esercizio di una potestà riservata dalla legge ad organi legislativi”). Lo stesso potrebbe dirsi, come rileva Gaetano Silvestri ${ }^{48}$, anche per la previsione secondo cui, ai fini della "carriera” del magistrato, sono presi in considerazione gli “esiti dei provvedimenti adottati”.

Un'ultima considerazione, in stretta connessione con quanto ho cercato finora di dire, può essere dedicata alla previsione, contenuta nella legge in questione, secondo cui "non può dar luogo a responsabilità disciplinare l'attività di interpretazione di norme di diritto in conformità all'art. 12 delle disposizioni sulla legge in generale" (art. 2, $6^{\circ}$ comma, lett. c), n. 11), dalla quale parrebbe, a contrario, doversi desumere che incorre in sanzione disciplinare il giudice che, nell'interpretare il diritto, non si attiene ai canoni previsti dalla citata disposizione. L'art. 12 delle preleggi stabilisce che "nell'applicare la legge non si può ad essa attribuire altro senso che quello fatto palese dal significato proprio delle parole secondo la connessione di esse e dalla

\footnotetext{
${ }^{46}$ Tra i rilievi svolti dal Presidente Ciampi nel rinviare la legge alle camere, uno era specificamente rivolto alla menomazione dei poteri del Consiglio superiore della magistratura risultante da alcune disposizioni della legge delega, per il fatto di collocare al centro di ogni procedura concorsuale la Scuola superiore della magistratura, struttura "esterna” al Csm e apposite commissioni, anch'esse "esterne” al Consiglio, concludendo che il sistema delineato nella legge sottoponeva sostanzialmente il Csm a un regime di vincolo che ne riduceva notevolmente i poteri definiti dall'art. 105 della Costituzione.

${ }^{47}$ Così SILVESTRI, Lo Stato senza principe. La sovranità dei valori nelle democrazie pluraliste, Torino, 2005,142 ss.

${ }^{48}$ SILVESTRI, La riforma dell'ordinamento giudiziario, in Giustizia più efficiente e indipendenza dei magistrati a garanzia dei cittadini, Milano, 2004, 129 ss.
} 
intenzione del legislatore. Se una controversia non può essere decisa con una precisa disposizione, si ha riguardo alle disposizioni che regolano casi simili o materie analoghe; se il caso rimane ancora dubbio, si decide secondo i principi generali dell'ordinamento giuridico dello stato".

A parte la difficoltà di determinare quando possa ritenersi che un magistrato non ha osservato le regole dell'art. 12 - il che, da un lato, viene a frustrare la finalità della “tipizzazione” dell'illecito disciplinare e, dall'altro, pare reintrodurre dalla finestra il divieto di interpretazione creativa - tutti coloro che, dopo l'entrata in vigore della Costituzione, si sono riferiti alla suddetta disposizione hanno concordato nel ritenere la stessa una sorta di vecchio arnese ormai inutilizzabile, rilevando ${ }^{49}$ come il riferimento ai principi generali (quindi in primis quelli costituzionali) viene, con la Costituzione, a subire una trasformazione totale, passando da una posizione di ultima ratio a presupposto cui tutte le norme debbono uniformarsi oppure ritenendo al massimo valutabili come meri consigli quelli contenuti nell'art. 12 delle preleggi e sottolineando come oggi, riferirsi a tale disposizione, significherebbe capire assai poco della giurisprudenza costituzionale oppure indurrebbe chi lo facesse a condannarla quasi in blocco ${ }^{50}$.

Se è vero che rimarrà sempre una illusione quella del legislatore di voler disciplinare (e quindi limitare) con legge l'attività interpretativa del giudice, se non altro per il fatto che anche quella legge dovrà essere interpretata, resta comunque quasi incredibile che una legge del 2005 si riferisca ancora ad una disposizione che la dottrina da molti anni considera assolutamente superata, inservibile e persino dannosa.

Data la connessione, che ho cercato di ricordare, sussistente tra il ruolo assegnato al giudice dall'ordinamento e la disciplina dell'organizzazione giudiziaria, la modificazione della legge sull'ordinamento giudiziario può essere fatta per conformare quest'ultima alle trasformazioni che ha subito il primo oppure allo scopo di contrastare il ruolo che quelle trasformazioni hanno assegnato all'autorità giudiziaria.

Qualora l'obiettivo perseguito dalla legge 150/2005 fosse stato il primo, credo si possa dire che essa avrebbe fatto riferimento ad un giudice assai diverso da quello previsto dall'attuale ordinamento e molto simile a quello che esisteva in Italia oltre cinquanta anni fa. Se invece, come pare più probabile, la finalità è stata quella indicata per seconda, la legge pare contrastare con il modello di giudice e di ordinamento giudiziario che si è venuto realizzando in attuazione dei principi fissati dalla Costituzione italiana e del diritto comunitario e pertanto viene a porsi inevitabilmente in contrasto con gli uni e con l'altro ${ }^{51}$.

${ }^{49}$ Cfr., tra gli altri, NATOLI, Alcune osservazioni su Costituzione cit., 280; PIZZORUSSO, Principali fattori evolutivi del sistema delle fonti del diritto italiano, in Il dialogo tra le corti. Principi e modelli di argomentazione, a cura di Navarretta e Pertici, Pisa, 2004, 20 ss.

${ }^{50}$ PALADIN, Le fonti del diritto cit., 104 ss.

${ }^{51}$ Per alcune riflessioni in ordine alla costituzionalità della disciplina ora contenuta nella l. 150/2005, v. ELIA, La riforma dell'ordinamento giudiziario: brevi considerazioni di un costituzionalista, in Questione giust., 2004, 625; DAL CANTO, ROMBOLI (cur.), Contributo al dibattito sull'ordinamento giudiziario, Torino, 2004; BALBONI, Ordinamento giudiziario e sistema costituzionale: alla ricerca del fondamento, in Questione giust., 2005, 671; SENESE, Modello di stato e riforma dell'ordinamento giudiziario, ivi, 2005, 131. 